\title{
Chest ultrasonography to detect lung involvement in Von Recklinghausen's disease
}

\author{
Maurizio Zanobetti • Beatrice Del Taglia • \\ Alberto Conti · Francesca Innocenti • \\ Riccardo Pini
}

Received: 10 January 2012 / Accepted: 20 March 2012/Published online: 13 April 2012

(C) SIMI 2012

A 44-year-old woman presented at the Emergency Department (ED) with cough and dyspnea started 2 days prior. She was an active smoker, and she had a history of neurofibromatosis type $1(\mathrm{NF})$ diagnosed about 20 years prior by biopsy of the first nodule on the lower lip without lung involvement. At presentation, the patient showed sinus tachycardia (110 beats/min), tachypnea (respiratory rate: 28 breaths/min), oxygen saturation $92 \%$ on room air, and body temperature $37.6^{\circ} \mathrm{C}$.

Physical examination revealed several café-au-lait maculae and several nodules on her trunk.

Pulmonary examination showed reduced breath sounds bilaterally associated with bilateral wheezes.

The 12-lead EKG demonstrated a normal sinus rhythm at a rate of $99 / \mathrm{min}$. Chest radiography revealed an image suggestive for pulmonary hyperlucency, without acute pulmonary consolidation and without bronchiectasis (Fig. 1). Laboratory tests revealed an elevated WBC $\left(12.9 \times 10^{9} / \mathrm{L}\right)$ and fibrinogen level $(479 \mathrm{mg} / \mathrm{dL})$. Arterial blood gas analysis showed hypoxia $\left(\mathrm{PaO}_{2} 66.4 \mathrm{mmHg}\right)$ with normal $\mathrm{pH}$ and $\mathrm{PaCO}_{2}$ values.

Based on the initial presentation, normal EKG, hypoxia and the presence of elevated inflammatory markers in the laboratory tests, pulmonary infection was considered.

Thus, oxygen therapy $(2 \mathrm{~L} / \mathrm{min})$ by nasal probes and antibiotics (Levofloxacin $750 \mathrm{mg}$, PO qd) were started. On

M. Zanobetti - B. Del Taglia - A. Conti - F. Innocenti - R. Pini Department of Critical Care Medicine and Surgery,

University of Florence, Florence, Italy

\section{Zanobetti ( $\square)$}

SOD Osservazione Breve Intensiva,

Azienda Ospedaliero-Universitaria Careggi,

Largo Brambilla, 3, 50134 Florence, Italy

e-mail: zanomau@libero.it the second day, the hemodynamic and respiratory status did not improve significantly. For this reason, we performed a chest ultrasonography that showed two sub-pleural hypoechogenic images, 0.5 and $0.7 \mathrm{~cm}$ in diameter, respectively, in the lower field of left lung with rear-wall reinforcement and a localized alveolar-interstitial syndrome (Fig. 2).

On the basis of the result of chest ultrasonography, we considered the clinical presentation as the lung involvement of NF. So, we added to the current therapy, methylprednisolone sodium succinate (60 mg daily, IV), and the association of beclomethasone, ipratropium bromide, and salbutamol $(0.8,0.25$ and $5 \mathrm{mg}$, respectively, nebulized bid). A significant improvement of respiratory status was noted in a few hours. The ultrasonographic findings were subsequently confirmed by a chest high resolution computed tomography (HRCT) that showed diffuse small rounded thin-walled lung cysts $(1 \mathrm{~cm})$, predominant in the lower left lobe. Same lung cysts had a diameter of $3.3 \mathrm{~cm}$ (Fig. 3). These abnormalities had a random distribution, prevalent on the sub-pleural side with paraseptal emphysema characteristics of neurofibromatosis. The chest HRCT also showed a moderately thickened appearance of the walls of some pyramids in basal bronchial branches, which could be compatible with bronchiectasis. This apparent discordance with the chest X-ray study can be explained by the fact that HRCT demonstrates higher sensivity than chest radiography in the detection of bronchiectasis.

Von Recklinghausen's disease or neurofibromatosis type 1 is an autosomal dominant dysplasia of ectoderm and mesoderm with a prevalence of 1 in 3,000. The typical characteristic is the presence of neurofibromas. The majority of patients have discrete benign neurofibromas within the dermis. Nodular neurofibromas arise from peripheral nerves at any site. Plexiform neurofibromas are 


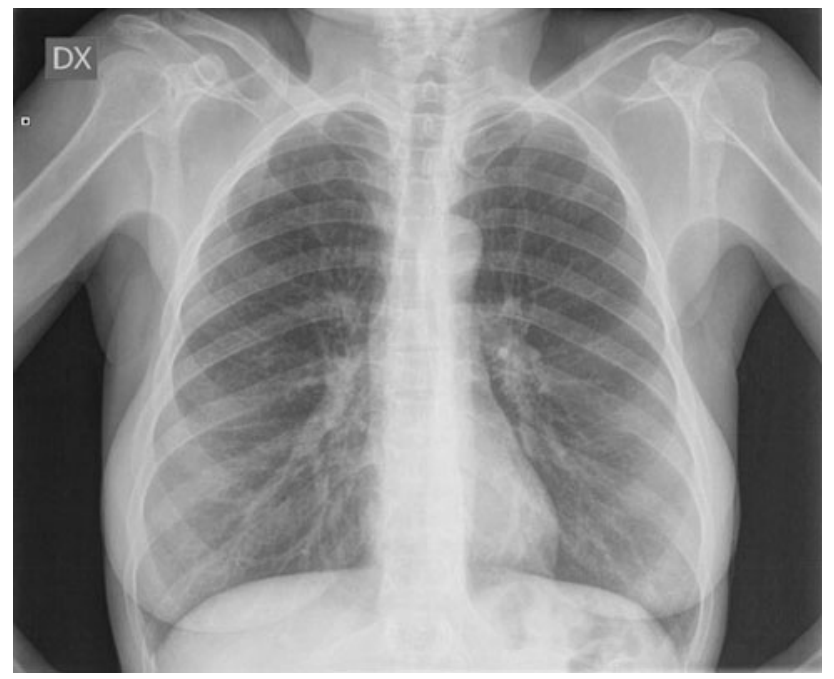

Fig. 1 Chest radiography showing abnormalities suggestive of pulmonary hyperinflation. Absence of acute parenchymal lung consolidations

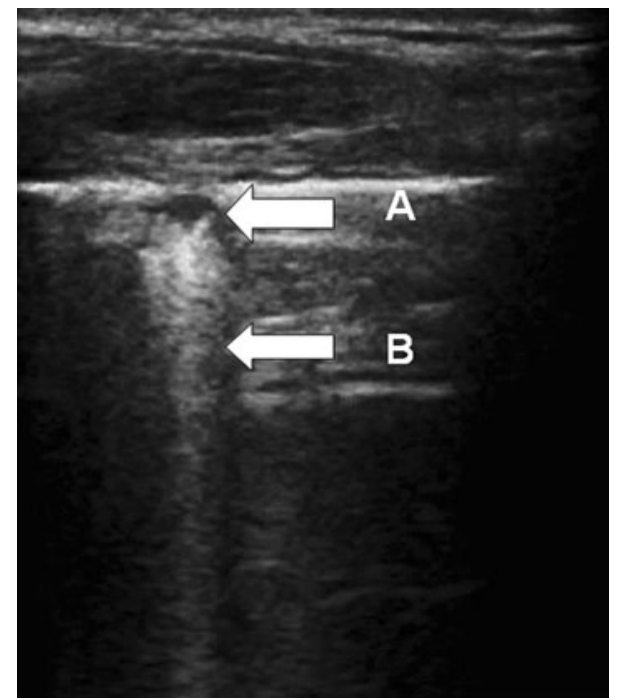

Fig. 2 Chest ultrasonography showing sub-pleural hypoechogenic image (a), in the lower field of left lung with rear-wall reinforcement and localized alveolar-interstitial syndrome (b)

usually congenital, and are present in $30 \%$ of the patients [1]. In about $2-16 \%$ of the patients, nodular and plexiform neurofibromas transform to malignant peripheral nerve sheath tumors [1]. Optic nerve gliomas are seen in $15 \%$ of the patients [1]. About $95 \%$ of the patients develop Lisch nodules [1].

In NF, the chest and lungs can be affected in several ways: cutaneous and subcutaneous neurofibromas on the chest wall, kyphoscoliosis, ribbon deformity of the ribs, and thoracic neoplasms [1]. An association of NF with diffuse lung disease (DLD) has been described, but its true prevalence and characteristics remain unclear and underestimated. The literature review reveals 64 adult patients

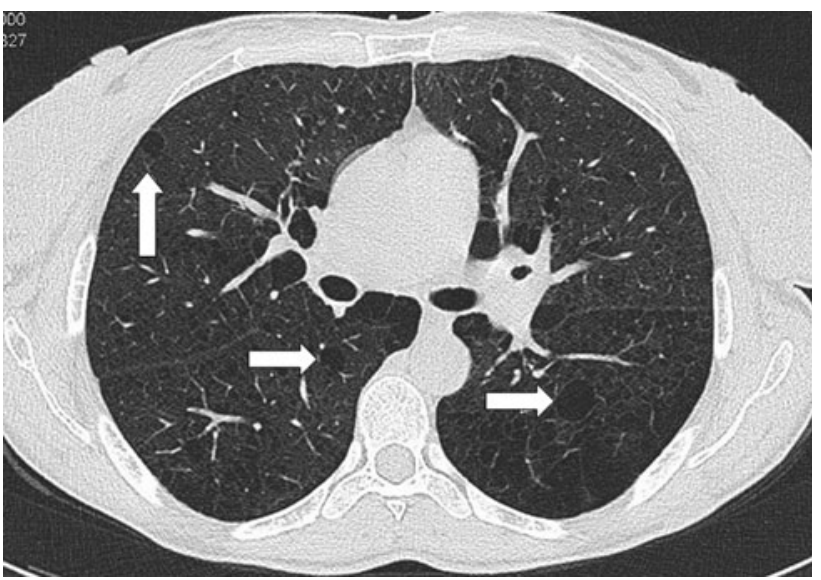

Fig. 3 Chest high resolution computer tomography showing diffuse small rounded thin-walled lung cysts (arrows), predominantly in the lower left lobe

affected by NF-DLD; mean age 50 years, $69 \%$ men. Common symptoms include dyspnea $(80 \%)$ and cough (32\%). Only $5 \%$ of the patients complain of chest pain and $11 \%$ are asymptomatic [2]. The average age and symptoms of ED presentation of our patient correlate with previous papers. Regarding the types of lung lesions detected by radiological examination, Zamora et al. [2] report that chest radiography demonstrates bullous lung disease in $73 \%$ of the cases, almost always in the upper lobes $(93 \%)$, and radiographic honeycombing sign in $13 \%$ of the cases. Chest HRCT reveals emphysema $(25 \%)$, cyst $(25 \%)$, ground-glass abnormality (37\%), bullas (50\%) and reticular abnormalities (50\%). Ryu et al. [3]. show a lack of evidence for an association between NF and parenchymal lung disease. Particularly, interstitial lung disease and bullas described in association with NF may represent, at least in part, smoking-induced manifestation [3]. In our case, chest radiography failed to show lung parenchymal abnormalities consistent with NF-DLD, whereas chest ultrasonography revealed the presence of sub-pleural abnormalities compatible with bullae confirmed by chest HRCT. To note, in our case, bullous lesions were predominant in the lower lobes while cases previously reported a prevalent upper lobes distribution. Unfortunately, since our patient was an active smoker, she could fall into the group with NF-DLD smoking-induced manifestation.

The diagnostic modalities currently available to demonstrate the lung involvement in NF are chest radiography and chest HRCT. In recent years, the use of chest ultrasonography in the evaluation of patients with dyspnea in the ED has been established, particularly demonstrating high concordance with radiography [4] and providing correct diagnoses of acute respiratory failure in $91 \%$ of the 
cases [5]. Our case suggests that chest ultrasonography may have higher diagnostic accuracy than chest radiography in the diagnosis of DLD even in patients with a rare pathology like NF.

Conflict of interest All authors have no conflict of interest to disclose.

\section{References}

1. Reynolds RM, Browning GG, Nawroz I, Campbell IW (2003) Von Recklinghausen's neurofibromatosis: neurofibromatosis type 1 . Lancet 361:1552-1554
2. Zamora AC, Collard HR, Wolters PJ, Webb WR, King TE (2007) Neurofibromatosis-associated lung disease: a case series and literature review. Eur Respir J 29:210-214

3. Ryu JH, Parambil JG, McGrann PS, Aughenbaugh GL (2005) Lack of evidence for an association between neurofibromatosis and pulmonary fibrosis. Chest 128:2381-2386

4. Zanobetti M, Poggioni C, Pini R (2011) Can chest ultrasonography replace standard chest radiography for evaluation of acute dyspnea in the ED? Chest 139:1140-1147

5. Lichtenstein DA, Meziere GA (2008) Relevance of lung ultrasound in the diagnosis of acute respiratory failure: the BLUE protocol. Chest 134:117-125 